\title{
Vertebral Height Growth Predominates Over Intervertebral Disc Height Growth in Adolescents With Scoliosis
}

\author{
Ian A. F. Stokes, PhD, ${ }^{*}$ and Luke Windisch, MASc $†$
}

Study Design. A cross-sectional study of spinal stereoradiographs of adolescents with scoliosis to measure growth.

Objective. To determine the relative contributions of the vertebral bodies and intervertebral discs to the increase in spinal length between T5 and L5, over the age range of 7.5-20 years.

Summary of Background Data. The progression of spinal deformity (scoliosis) is associated with skeletal growth, but the relative roles of asymmetrical growth and remodeling of the vertebrae and discs during adolescent growth are unclear.

Methods. An existing database of 406 spinal stereoradiographic studies of 188 adolescents with idiopathic scoliosis, aged between 7.5 and 20 years, was used to measure the heights of vertebral bodies and intervertebral discs, and the summation of both (spinal length).

Results. Spinal length was observed to increase from about 250 to $350 \mathrm{~mm}$ over this range of ages. Spinal growth was associated with an increase in vertebral height after age 10 years, with minimal if any increase in disc height. The contribution of vertebral and discal height was estimated to be about 17 and $8 \mathrm{~mm}$ per year, respectively, at age 7.5 years, but discal height growth was estimated to be effectively zero after age 12 years.

Conclusion. Spinal growth of patients with scoliosis aged between 10 and 20 years occurs almost exclusively by height increases in the vertebrae, not the discs.

Key words: growth, adolescence, skeletal maturity, vertebra, intervertebral disc, scoliosis. Spine 2006;31:1600-1604

Growth occurs in the spine during adolescence, as evidenced by the increase in sitting height. ${ }^{1,2}$ The highest annual growth in sitting height in a Taiwanese population $^{3}$ was $61 \mathrm{~mm}$ in boys and $63 \mathrm{~mm}$ in girls, at age 8.5 years, with a second peak of $46 \mathrm{~mm}$ in boys at age 12.5 year and $32 \mathrm{~mm}$ in girls at age 11.5 years. Sitting height increases are not necessarily synchronized with leg growth. ${ }^{4,5}$ Howell et $a l^{6}$ reported continued growth (about $14 \mathrm{~mm}$ increase in sitting height) after skeletal maturity, indicating that the spine continues to grow after cessation of limb growth.

From the *Department of Orthopaedics and Rehabilitation, University of Vermont, Burlington, VT, and +École Polytechnique de Montréal, Institut de génie biomédicale, Montréal, Quebec, Canada.

Acknowledgment date: June 14, 2005. First revision date: August 12, 2005. Acceptance date: September 6, 2005.

The manuscript submitted does not contain information about medical device(s)/drug(s).

Federal and Institutional funds were received in support of this work. No benefits in any form have been or will be received from a commercial party related directly or indirectly to the subject of this manuscript.

Address correspondence and reprint requests to Ian A. F. Stokes, PhD, Department of Orthopaedics and Rehabilitation, University of Vermont, Stafford Hall, Burlington, VT 05405-0084; E-mail: Ian.Stokes@uvm.edu
Human vertebrae grow in height by a mechanism similar to that in long bones, by endochondral ossification in growth plates adjacent to the discs, and they increase in diameter by appositional growth. ${ }^{7}$ Growth of the neural canal dimensions, including increase in interpedicular distance, is near complete in early childhood. ${ }^{8}$ Sustained mechanical loading can modulate the growth in height. ${ }^{9}$

In contrast to the growth of bones that occurs locally in specialized growth plates and apophyses, discs probably grow by a distributed mechanism of cell proliferation and matrix synthesis, although little is apparently known about postnatal growth of the discs. ${ }^{10,11}$ Human vertebrae are unusual because they lack the ossified and vascularized secondary centers of ossification (epiphyses) that form between the growth plate and discs of most other vertebrates. This may place the discs at additional metabolic risk during skeletal growth because they must compete with the growth plates for the nutrition that originates in the vertebral body's vasculature. Antoniou et $a l^{12}$ reported a steady decline in the rate of matrix synthesis in the disc over all decades of life, with collagen denaturation occurring at a relatively steady rate in the age range of 5-25 years. Rat discs appear to continue growing throughout the first 18 months of life, ${ }^{11}$ but these radiographic measurements had limited accuracy because of difficulties with the radiographic method, as well as acute changes in disc height with in vivo loading conditions, etc.

It is not clear how the differential growth of the spine is regulated in relationship to the coordinated control of skeletal growth. Scoliosis is apparently a result of inadequate control of spinal growth. The deformity progresses most rapidly during adolescent growth, ${ }^{13}$ and residual growth is a risk factor for scoliosis progression. ${ }^{14}$ There is evidence that adolescents with idiopathic scoliosis have an earlier growth spurt. ${ }^{15}$ The mechanism of progression of postnatal skeletal growth deformity such as scoliosis is often attributed to the "Hueter-Volkmann Law" of mechanically modulated endochondral growth in a "vicious cycle."9,16 Therefore, scoliosis progression slows or ceases at skeletal maturity. ${ }^{17}$ However, the simultaneous apparent acceleration of progression of intervertebral disc deformity at puberty and slowing at skeletal maturity occurs in the absence of any known dramatic changes in intervertebral disc metabolism in the second decade.

Improved treatment of spinal deformity progression during adolescent growth may result from a better understanding of how spinal growth occurs in vertebrae and intervertebral discs. The present study made use of a preexisting set of 3-dimensional (3-D) spinal measure- 
ments from stereoradiographs of patients attending a scoliosis clinic. ${ }^{18}$ The objective was to identify the relative contributions of the height increases in discs and vertebrae to the elongation of the spinal column.

\section{Methods}

Vertebral and discal heights measured from 406 stereoradiographs ${ }^{18}$ of 188 patients with a diagnosis of idiopathic adolescent or juvenile idiopathic scoliosis were studied. The stereoradiographs had been made during the period of 1981-1986. Data were included in the present study for patients $7.5-20$ years old, before any surgery. There were 33 boys and 155 girls. Of the 406 radiographs, 98 were recorded as being of patients undergoing brace treatment at radiography. According to the records, 8 radiographs were of patients while wearing their brace. Of the 188 patients, 104 had 1 radiographic record, 32 had 2, and 52 had more than 3 (maximum 11) radiographic studies. Distribution of the ages of these patients at stereoradiography are shown in Figure 1. The Cobb angle of the largest curve had been recorded for 319 radiographs. Mean Cobb angle was $24^{\circ}$ (range $3^{\circ}-75^{\circ}$, mode $20^{\circ}$ ).

The patients had been radiographed in a controlled standing posture, with supports contacting the anterior superior iliac spines and clavicles, with arms to the sides, to minimize patient motion during radiography. There were 2 radiographic projections made with a $3 \mathrm{~m}$ film-to-focus distance, using 36-in (914 $\mathrm{mm}$ ) cassettes, with low-dose intensifying screen and film combinations. Posteroanterior and oblique views were made. The oblique view used an $\mathrm{x}$-ray tube either at $20^{\circ}$ on the patient's right side or $15^{\circ}$ above the horizontal.

For each stereo reconstruction of the spine, vertebral landmarks (vertebral endplates and bases of pedicles) had been previously identified, marked, and digitized from each radiograph according to the methods described ${ }^{18}$ to obtain the 3-D coordinates of each landmark. The height of each vertebra and disc was determined as the Pythagorean distance between the 3-D coordinates of the corresponding endplate centers. Spinal length was defined as the sum of the heights of all vertebrae and discs. Length contributions of the vertebrae and intervertebral discs were defined as the sum of all vertebral and disc heights, respectively. Although all vertebral landmarks from T1 to L5 had been measured, the vertebrae and discs above T5 were omitted from this study because they were frequently unclear on the original films as a result of exposure or projectional problems.

For each of the measurements (i.e., spinal length, total vertebral height, and total disc height), regression analysis determined a nonlinear mathematical relationship with age. Because growth relationships are asymptotic to a final dimension, logistic regression analysis ${ }^{19}$ was used to obtain an analytical curve fit to observed spinal dimensions and patient age, using the following equation:

$$
h=a /\left(1+e^{(m-y) / s}\right)
$$

where $\mathrm{h}=$ summated height $(\mathrm{mm})$ of the vertebrae or discs or both; $y=$ age of patient (years); and a, $\mathrm{m}$, and s are parameters in the curve fit.

The parameter "a" represents the asymptote of the curve, thus, it is a measure of the final height (of the spine, and its components), and values of " $\mathrm{m}$ " and "s" provide a fit to the amount of growth and rate of growth, respectively.

The parameters "a," " $\mathrm{m}$," and "s" were estimated by leastsquares fit to the observed data points, using the function "fminsearch.m" in the Matlab programming language (Mathworks, Natick, MA). Subsequently, estimates of the growth (increase in height) per year were derived from the slope (first derivative) of the nonlinear regression. A linear regression analysis was used to identify significant trends in vertebral height, disc height, and spinal length increases with time.
Figure 1. Histogram of the ages of patients when the 406 stereoradiographs were made.

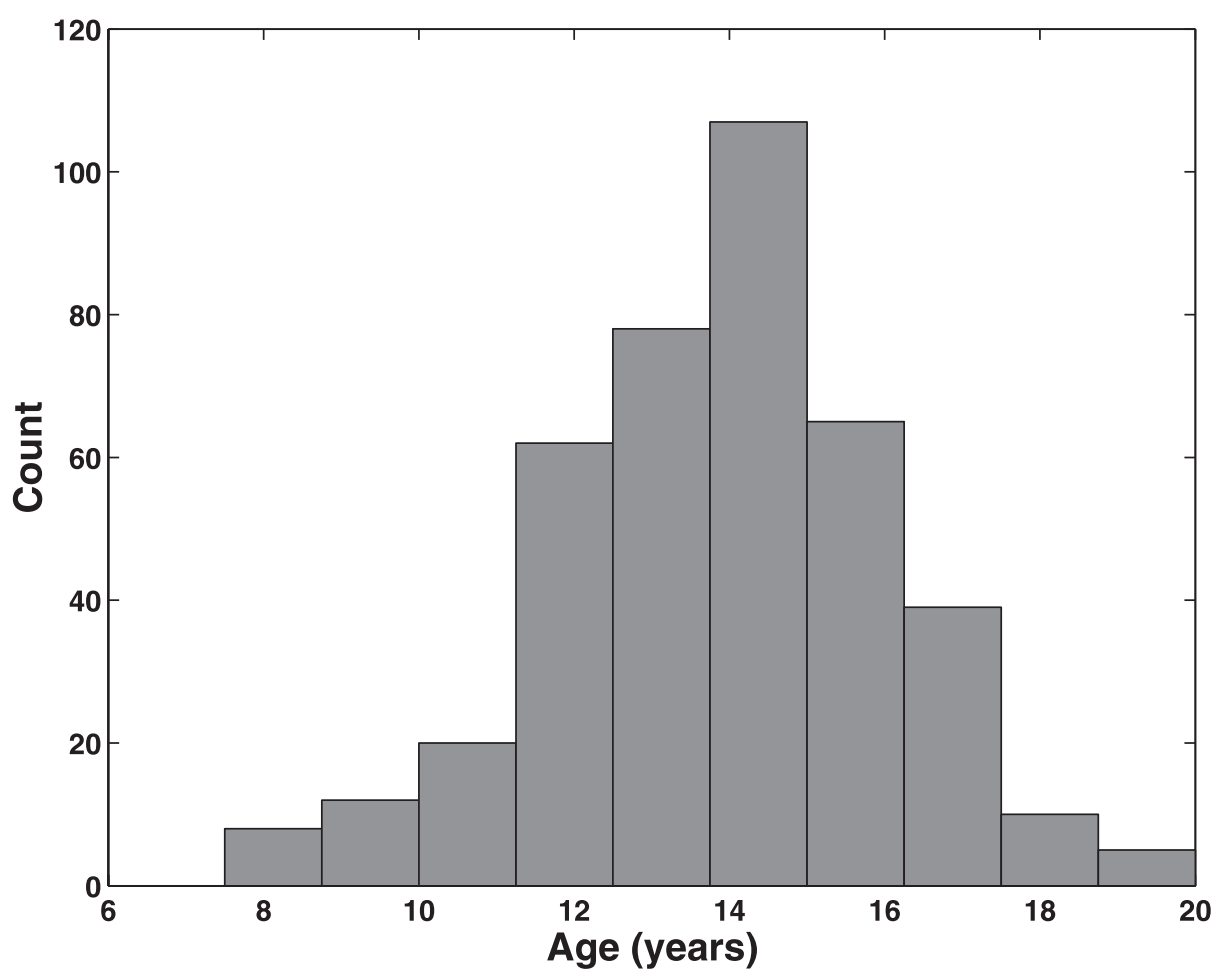


Figure 2. The measured spinal dimensions plotted against age. The solid lines correspond to curves fitted to the observed data by logistic regression (see equation in the "Methods" section). $x$ indicates total spinal length T5-L5; squares show summated heights of vertebrae; and circles illustrate summated heights of intervertebral discs.

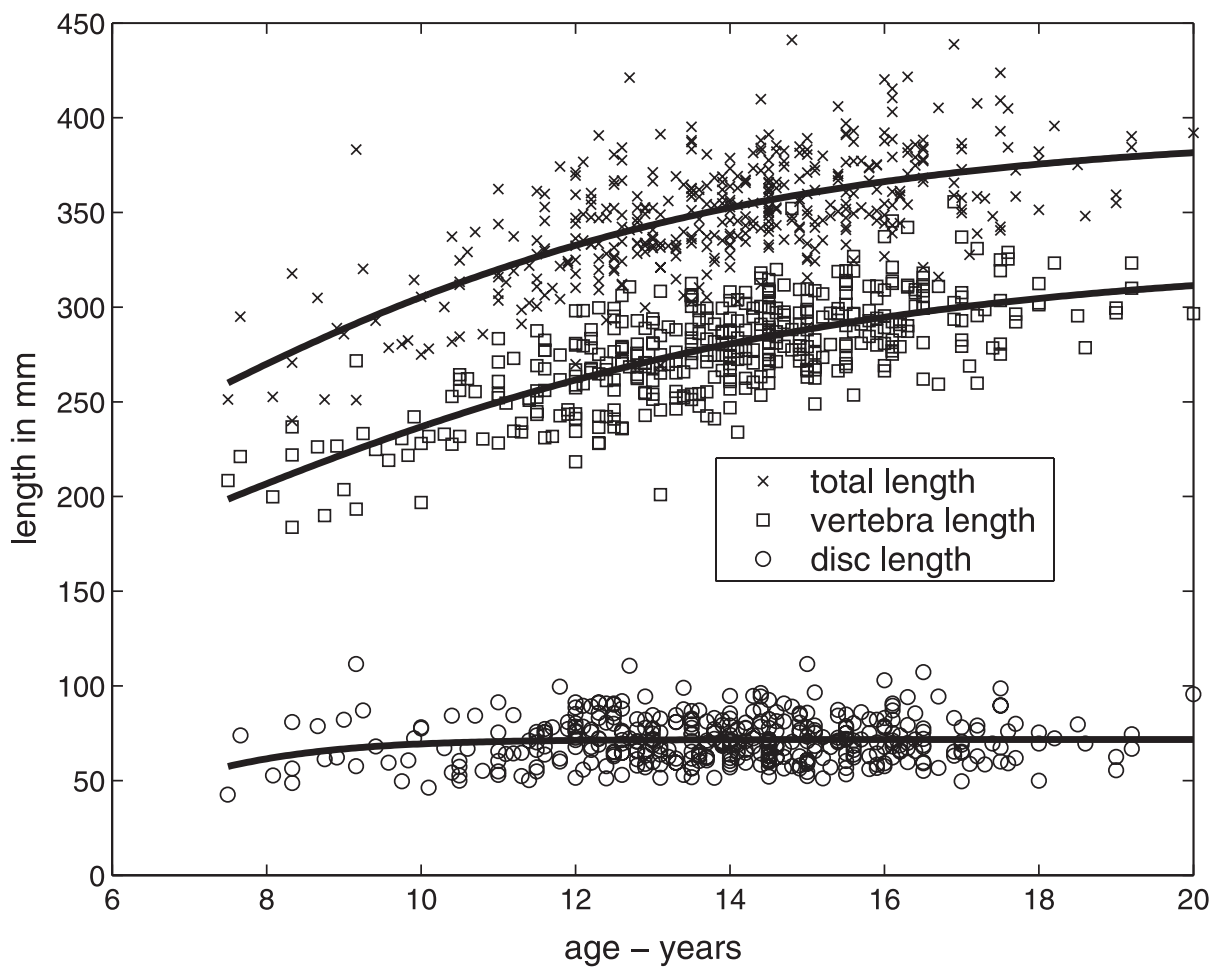

\section{Results}

The total spinal length (T5-L5) increased in this population from approximately $250 \mathrm{~mm}$ at age 7.5 years to $380 \mathrm{~mm}$ at age 20 years. There was little contribution from intervertebral disc height increase to this spinal growth after about age 10 years (Figure 2). In Figure 2, the solid lines that correspond to the logistic regression fit to observed data indicate that vertebral growth continued during the second decade but that there was a decline to apparently negligible rates of disc height increase during the same period. The parameter values obtained from the logistic regressions for each curve are listed in Table 1. Based on linear regression analysis, there was a statistically significant $(P<0.001)$ trend of increasing total vertebral height with age, while no such significant increase existed for disc height. The growth in spinal length estimated by taking the first derivative of the height-age nonlinear regression relationship declined from about $20 \mathrm{~mm}$ per year to about $3 \mathrm{~mm}$ per year, over the age range of 7.5-20 years (Figure 3 ). Before age 10

Table 1. Values of Parameters Obtained by Nonlinear Regression (see equation in the "Methods" section) of Measured Spinal Length, and the Contributions of Vertebrae and Discs Versus Age

\begin{tabular}{lccc}
\hline Parameters & $\begin{array}{c}\text { Spinal Length } \\
\text { (T5-L5) }\end{array}$ & $\begin{array}{c}\text { Sum of } \\
\text { Vertebral Heights }\end{array}$ & $\begin{array}{c}\text { Sum of } \\
\text { Discal Heights }\end{array}$ \\
\hline a (asymptote) & $392.27 \mathrm{~mm}$ & $325.0 \mathrm{~mm}$ & $71.69 \mathrm{~mm}$ \\
s (y) & 4.31 & 4.66 & 1.25 \\
m (y) & 4.59 & 5.39 & 5.75 \\
\hline
\end{tabular}

years, the height increases of both vertebrae and discs contributed to this spinal growth, but, subsequently, after age 10 years, the estimated contribution of disc height growth declined to a negligible rate.

The 308 radiographs of patients not undergoing treatment were analyzed separately by differentiation of the logistic regression fits to determine the spinal growth rate. The growth rate of the spine in this subset declined from $15 \mathrm{~mm}$ per year at age 7.5 years to $4.2 \mathrm{~mm} / \mathrm{y}$ at age 20 years. Thus, it was slower at age 7.5 years and more rapid at age 20 years than in the full sample. Assuming that the braced patients were those with more progressive scoliosis, this suggests that the subset (untreated patients) had a later growth spurt than the braced patients, consistent with the observation of Hagglund et al. ${ }^{15}$ In the untreated subset, there was minimal growth (less than $2 \mathrm{~mm} / \mathrm{y}$ ) attributable to the discs at all ages.

\section{Discussion}

The aim of this study was to determine the relative contributions of height increases of the vertebra and discs to growth in spinal length during adolescence in patients with scoliosis. It was found that there was little to no intrinsic growth in the height of the intervertebral discs after age 10 years, therefore, almost all spinal growth occurred in the vertebrae. The regression analyses included multiple (longitudinal) observations of some patients, and also included both male and female patients. This cross-sectional study averaged the spinal growth of many patients, and probably "smoothed-out" individual growth spurts, thereby underestimating peak growth velocity. The measurements corresponded to the part of the 
Figure 3. Growth rates $(\mathrm{mm} / \mathrm{y})$ obtained as the slope (first derivative) of the nonlinear regression curve fits, as shown in Figure 2.

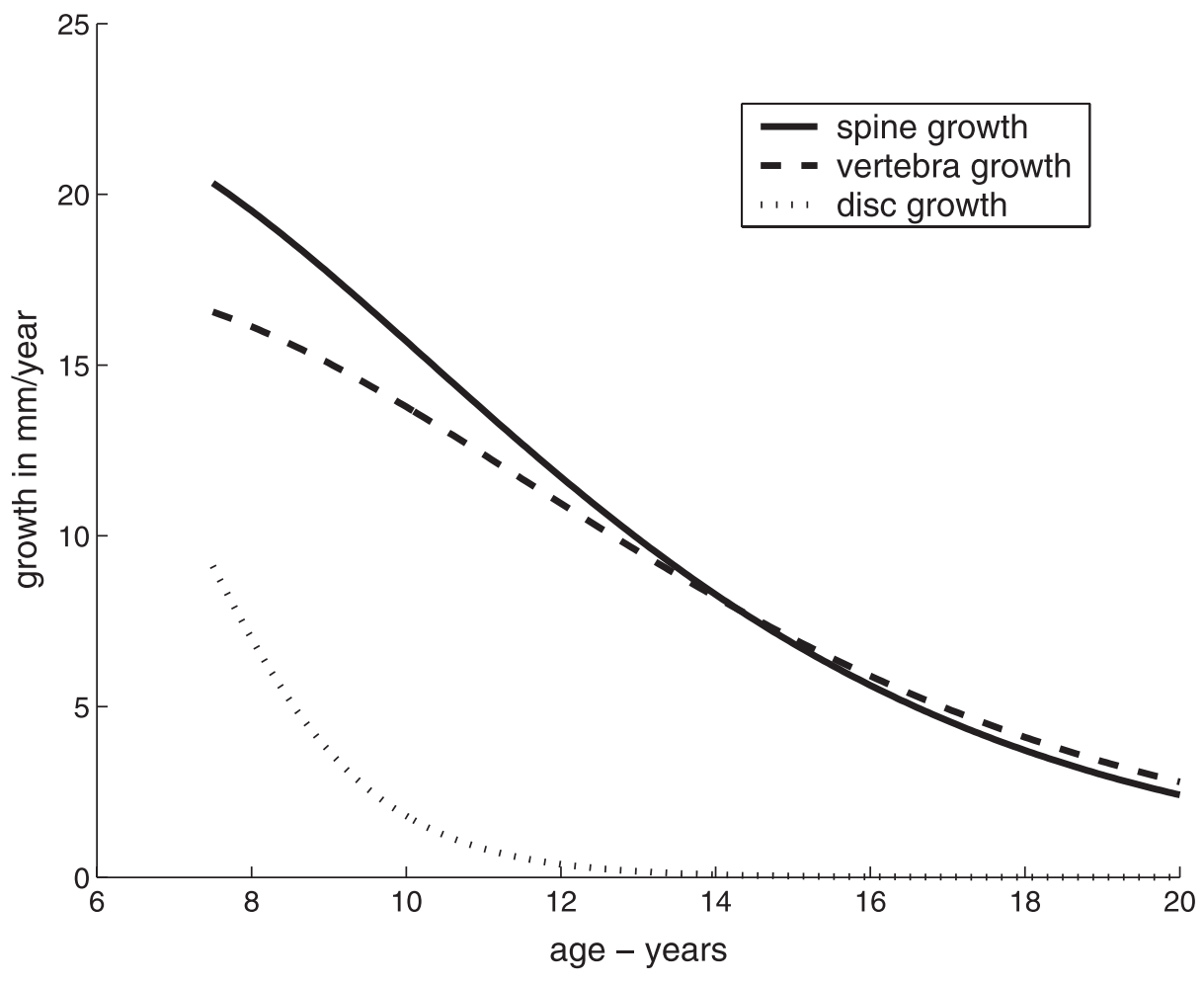

spine between T5 and L5, and the vertebral and discal height measures were based on distances between points at the centers of the vertebral endplates.

The findings of this study were obtained from a group of patients with idiopathic scoliosis. However, the overall finding of growth occurring predominantly in the vertebrae can probably be generalized to adolescents without scoliosis, although the timing of the growth spurt may differ. ${ }^{15}$ There is little gross disturbance of overall skeletal growth and sitting/standing ratio in scoliosis. ${ }^{20}$ About a quarter of the radiographs were of patients undergoing brace treatment. Although braces may alter the evolution of the Cobb angle (vertebral plus disc wedging), they probably do not substantially alter overall spinal loading, or longitudinal growth. In addition, the duration of brace wearing before radiographs being taken was probably insufficient to make a significant difference in accumulated growth. It is not known whether the wedging of vertebrae and discs in scoliosis affects their overall height as measured here along the central axis of the spine.

There was a small number of observations at the extremes of the age range studied here. Therefore, true values for the increase in disc heights up to age 10 years as well as the apparent continued growth of the spine at age 20 years are not certain. Howell et al ${ }^{6}$ reported continued spinal growth of about $4 \mathrm{~mm}$ per year for 3 years after apparent skeletal maturity. A similar rate of growth after age 17 years was observed in the present study. Howell et $a l^{6}$ considered that this continued spinal growth might be the cause of continued progression of scoliosis among young adults. The present study indicates that the spinal growth occurring during progression of adolescent scoliosis occurs almost exclusively in the vertebrae, although it is possible that discal wedging can progress in the absence of growth in the height of the discs.

\section{- Key Points}

- Growth in the spine between the ages of 10 and 20 years occurs almost exclusively by height increases in the vertebrae, not the discs.

- The estimated population average growth in spinal length declined from about $20 \mathrm{~mm}$ per year to about $3 \mathrm{~mm}$ per year over the age range of 7.5-20 years.

- The absence of disc growth implies that the mechanism of progressive wedging during adolescent growth in scoliosis differs between vertebrae and discs.

\section{References}

1. Aldegheri R, Agostini S. A chart of anthropometric values. J Bone Joint Surg Br 1993;75:86-8.

2. Nicolopoulos KS, Burwell RG, Webb JK. Stature and its components in healthy children, sexual dimorphism and age related changes. J Anat 1985;141:105-14.

3. Lee TS, Chao T, Tang RB, et al. A longitudinal study of growth patterns in schoolchildren in one Taipei District. II: Sitting height, arm span, body mass index and skinfold thickness. J Chin Med Assoc 2005;68:16-20.

4. Butler GE, McKie M, Ratcliffe SG. The cyclical nature of prepubertal growth. Ann Hum Biol 1990;17:177-98.

5. Diméglio A. Growth in paediatric orthopaedics. In: Morrissy RT, Weinstein SL, eds. Lovell and Winter's Pediatric Orthopaedics. 5th ed. Philadelphia, PA: Lippincott Williams and Wilkins; 2001:33-98.

6. Howell FR, Mahood JK, Dickson RA. Growth beyond skeletal maturity. Spine 1992;17:437-40.

7. Dickson RA, Deacon P. Annotation: Spinal growth. J Bone Joint Surg Br 1987;69:690-2.

8. Tulsi RS. Growth of the human vertebral column. An osteological study. Acta Anat (Basel) 1971;79:570-80. 
9. Stokes IAF, Spence H, Aronsson DD, et al. Mechanical modulation of vertebral body growth: implications for scoliosis progression. Spine 1996;21:1162-7.

10. Urban JPG, Roberts S. Development and degeneration of the intervertebral discs. Mol Med Today 1995;1:329-35.

11. Hulse Neufeld J, Haghighi P, Machado T. Growth related increase in rat intervertebral disc size: A quantitative radiographic and histologic comparison. Lab Anim Sci 1990;40:303-7.

12. Antoniou J, Steffen T, Nelson F, et al. The human lumbar intervertebral disc: Evidence for changes in the biosynthesis and denaturation of the extracellular matrix with growth, maturation, ageing, and degeneration. J Clin Invest 1996;98:996-1003.

13. Little DG, Song KM, Katz D, et al. Relationship of peak height velocity to other maturity indicators in idiopathic scoliosis in girls. J Bone Joint Surg Am 2000;82:685-93.
14. Lonstein JE, Carlson JE. The prediction of curve progression in untreated idiopathic scoliosis during growth. J Bone Joint Surg Am 1984;66:1061-71.

15. Hagglund G, Karlberg J, Willner S. Growth in girls with adolescent idiopathic scoliosis. Spine 1992;17:108-11.

16. Roaf R. Vertebral growth and its mechanical control. J Bone Joint Surg Br 1960;42:343-9.

17. Risser JC. Scoliosis: Past and present. J Bone Joint Surg Am 1964;46:167-99.

18. Stokes IAF, Bigalow LC, Moreland MS. Three-dimensional spinal curvature in idiopathic scoliosis. J Orthop Res 1987;5:102-13.

19. Tanner JM, Whitehouse RH, Marubini E, et al. The adolescent growth spurt of boys and girls of the Harpenden growth study. Ann Hum Biol 1976;3: $109-26$.

20. Willner S. The proportion of legs to trunk in girls with idiopathic structural scoliosis. Acta Orthop Scand 1975;46:84-9. 